H. YAMADA

KODAI MATH. J.

2 (1979), 346-361

\title{
BOUNDARY VALUE PROBLEMS FOR REAL LINEAR PARTIAL DIFFERENTIAL EQUATIONS OF FIRST ORDER
}

\author{
BY HARUKI YAMADA
}

\section{$\S 1$. Introduction.}

Let $\Omega \subset R^{n}$ be a bounded connected open set with $C^{\infty}$-boundary $\Sigma=\partial \Omega$. We consider the following equation.

$$
L u=\sum_{\imath=1}^{n} b^{\imath}(x) \frac{\partial u}{\partial x_{\imath}}+c(x) u=f(x),
$$

where $b^{2}(x), c(x), f(x) \in C^{\infty}(\bar{\Omega})$ and all the functions are assumed to be real valued. We shall concern the problem: When, for suitably chosen closed subset $\Gamma \leqq \Sigma$, is the following statement true ?

For any $f \in C^{\infty}(\bar{\Omega})$ and $g \in C^{\infty}(\Gamma)$, there is one and

$\left[W_{m}\right] \quad$ only one solution $u \in C^{m}(\bar{\Omega})$ of

$$
L u=f,\left.\quad u\right|_{\Gamma}=g,
$$

where $m=1,2, \cdots \infty$. Though, as is well known, the problem of solving first order partial differential equation (1.1) is reduced to solving system of first order ordinary difierential equations

$$
\frac{d x_{\imath}}{d t}=b^{\imath}(x), \quad \imath=1,2, \cdots n,
$$

it is only a local result and our problems are related to global behavior of solution curves of (1.2). Our problems are also related to the first boundary value problems for second order equations with non-negative characteristic form investigated by Kohn-Nierenberg [2], Oleinik [3] and others. Though our equation (1.1) is a very special case of these degenerate second order equations, some of the difficulties in these problems are related to our problems and some meanings of conditions imposed to these problems are clarified by our settings.

We can pose the problem "under what conditions for $L, \Omega$ and $\Gamma$, is the statement $\left[W_{m}\right]$ true ?" for general second order equations with not necessary non-negative characteristic forms. We shall not concern this problem in this note but show some simple examples at the end of this note.

Received June 2, 1978 
I wish to express my gratitude to Professor Yoshikazu Hirasawa for his constant encouragements and valuable advices.

\section{$\S 2$. The general case.}

We use the following notation.

$$
L^{0} u=\sum_{\imath=1}^{n} b^{\imath}(x) \frac{\partial u}{\partial x_{\imath}} .
$$

At first we note a result concerning $C^{\infty}$-solvability of the first order equations.

Proposition 2.1. The followng conditions are equivalent.

a) $L^{0} C^{\infty}(\bar{\Omega})=C^{\infty}(\bar{\Omega})$,

b) $\left(L^{0}+c\right) C^{\infty}(\bar{\Omega})=C^{\infty}(\bar{\Omega})$ for any $c(x) \in C^{\infty}(\bar{\Omega})$,

c) There is a function $\varphi \in C^{\infty}(\bar{\Omega})$ such that $L^{0} L^{0} \varphi>0$ on $\bar{\Omega}$,

d) There is no complete integral curve of $L^{0}$ contaned in $\bar{\Omega}$.

For a simple proof we refer to [1], p. 212.

Remark. From the condition c) it follows that there are no critical points of the vector field

$$
b(x)=\left(b^{1}(x), \cdots, b^{n}(x)\right)
$$

in $\bar{\Omega}$. The relation between global solvability of (1.1) and periodic orbits of (1.2) seems not to be clarified yet.

Before investigating our problem, we note some examples which are compared with the results concerning solvability of boundary value problems.

EXAMPLE 2.1. $L^{0} u=\frac{\partial u}{\partial x}, \Omega=\left\{(x, y) ; 1<x^{2}+y^{2}<4\right\}$. Then $\left.c\right)$ is valid for $\varphi=x^{2}$ and $\left(L^{0}+c\right) u=f$ is globally solvable on $\bar{\Omega}$.

EXAMPLE 2.2. $L^{0} u=x \frac{\partial u}{\partial x}+y \frac{\partial u}{\partial y}$ and $\Omega$ is the same as above. Then c) is valid for $\varphi=x^{2}+y^{2}$ and $\left(L^{0}+c\right) u=f$ is globally solvable.

EXAmple 2.3. $L^{0} u=y \frac{\partial u}{\partial x}-x-\frac{\partial u}{\partial y}$ and $\Omega$ is the same as above. Then $\mathrm{d}$ ) is not valid since the vector field $b(x, y)=(x,-y)$ has an integral curve $x^{2}$ $+y^{2}=3 / 2$ completely contained in $\Omega$.

Now we shall concern solving the boundary value problem. At first we consider the problem: What conditions on $\Gamma$ must be imposed to ? Put

$$
\begin{aligned}
& \Sigma_{+}=\left\{x \in \Sigma ; \sum_{i=1}^{\nu} b^{\imath} \nu_{\imath}>0\right\}, \quad \Sigma_{-}=\left\{x \in \Sigma ; \sum_{i=1}^{\nu} b^{\imath} \nu_{\imath}<0\right\}, \\
& \Sigma_{0}=\left\{x \in \Sigma ; \sum_{i=1}^{\nu} b^{\imath} \nu_{\imath}=0\right\},
\end{aligned}
$$


where $\nu(x)=\nu=\left(\nu_{1}, \cdots, \nu_{n}\right)$ is the exterior unit normal vector of $\Sigma$ at $x \in \Sigma$.

Proposition 2.2. Suppose $b(x) \neq 0$ on $\bar{\Omega}$. Further, suppose that there are no periodic orbits of (1.2) which are completely contanned in $\bar{\Omega}$. Then for $\left[W_{m}\right]$ to be true it is necessary to take $\Gamma=\bar{\Sigma}_{+}$or $\Gamma=\bar{\Sigma}_{-}$.

Proof. Let $x(t)=\left(x_{1}(t), \cdots x_{n}(t)\right)$ be a solution of

$$
\frac{d x_{\imath}}{d t}=b^{2}(x), \quad \imath=1, \cdots, n \text {. }
$$

When we write, for a solution $u \in C^{m}(\bar{\Omega})$ of (1.1), $U(t)=u(x(t)), F(t)=f(x(t))$, $C(t)=c(x(t))$, we have

$$
\frac{d U(t)}{d t}+C(t) U(t)=F(t)
$$

Accordingly, when we prescribe the value $U(0)=u\left(x^{0}\right)$ at $t=0\left(x(0)=x^{0}\right)$, the solution of (2.2) has the form

$$
U(t)=e^{-h(t)}\left(U(0)+\int_{0}^{t} F(t) e^{h(t)} d t\right)
$$

along the curve $C: x=x(t)$, here $h(t)$ is defined by the solution of

$$
\frac{d h}{d t}=C(t), \quad h(0)=0 .
$$

Now we return to the problem

$$
L u=f,\left.\quad u\right|_{\Gamma}=g .
$$

We first note that for $\left[W_{m}\right]$ to be valid, $\Gamma$ must be the closure $\overline{\dot{ }} \dot{\Gamma}$ of its interior points $\stackrel{\circ}{\Gamma}$. In fact, if $\Gamma$ contain $x_{0} \in \Gamma-\overline{\dot{\Gamma}}$ there is a neighbourhood $\omega$ of $x_{0}$ such that $\omega-\Gamma \cap \omega$ is dense in $\omega$. Thus when we suppose that $\left[W_{m}\right]$ is true, the value of $u$ on $\omega-\Gamma \cap \omega$ must be determined by the data on $\Gamma$. But when we vary the data on $\Gamma \cap \omega$, the solution may become discontinuous on the set of the trajectry of flow from $\omega$ determined by (1.2). Thus $\Gamma$ must be the closure of its inner points. Next suppose that the data are given on $\Gamma \subsetneq \bar{\Sigma}_{+}$. Since $\Sigma_{+}$is an open set in $\Sigma$ we can take $x^{0} \in{\overline{\Sigma_{+}}}_{+}-\Gamma$ such that for some neighbourhood $\omega$ of $x_{0}, \omega \subset \bar{\Sigma}_{+}-\Gamma$. The union of trajectries of flows $x(t)$ which start from $\omega$ make an open set $U$ in $\Omega$ and on this set we cannot determine the value of $u(x)$ only from $f$ and $g$. Thus, for the uniqueness of the solution, it is necessary that $\Gamma \leftarrow \Gamma_{+}$.

Next, suppose that $\bar{\Sigma} \varsubsetneqq \Gamma$. We first note that $\Gamma$ must not include any inner points of $\Sigma_{0}$. In fact if $x_{0} \in \Gamma$ is an inner point of $\Sigma_{0}$, by transforming the coordinate system near $x_{0}$ such that

$$
\Sigma: x_{n}=0 \text {, }
$$


we have $b(x)=\left(b^{1}(x), \cdots, b^{n-1}(x), 0\right)$ on $\Sigma$ near $x_{0}$. From this it follows that for the flow $x(t)$ through $x_{0}$ at $t=0$, there is an open interval $(a, b)(a<0<b)$ such that $x(t) \in \Gamma$ for all $t \in(a, b)$. This contradicts the fact that we may take any boundary data on $\Gamma$. Thus if $\bar{\Sigma}_{+} \subsetneq \Gamma, \Gamma$ must contain an innerp oint $x_{1}$ of $\bar{\Sigma}_{-}$. Take a neighbourhood $\omega$ of $x_{1}$ such that $\omega \subset \Gamma \cap \Sigma_{-}$. Let $U \subset \Omega$ be the set of trajectries through $\omega$ and let $\tilde{\omega}=\bar{U} \cap \Sigma-\omega$. Since $\tilde{\omega}$ contain inner points of $\Sigma$, it may not happen that $\widetilde{\omega} \subset \Sigma_{0}$. Thus $\widetilde{\omega} \cap \Sigma_{+} \neq \phi$ and from the assumption $\bar{\Sigma}_{+} \subset \Gamma$, the contradiction follows. That the case $\Gamma \cap \bar{\Sigma}_{+} \neq \phi$ and $\Gamma \cap \bar{\Sigma}_{-} \neq \phi$ is impossible follows in a similar way.

In the above case, it is not required any conditions concerning $c(x)$. Next we consider the case when $c(x)>0$ in $\bar{\Omega}$ and $b(x)$ is arbitrary.

Proposition 2.3. Suppose that $c(x)<0$ on $\bar{\Omega}$ (resp. $c(x)>0)$. Then for $\Gamma=\bar{\Sigma}_{+}$ (resp. $\bar{\Sigma}_{-}$), the solution of (2.4) is unique.

Proof. It is sufficient to prove that for any solution $u \in C^{\infty}(\bar{\Omega})$ of $(2.4)$, we have

$$
\max _{\bar{\Omega}}|u| \leqq \max \left\{\sup _{\Omega}|f / c|, \max _{\bar{\Sigma}+}|g|\right\}=M .
$$

If we put $v_{ \pm}=M \pm u$, since $M \geqq \sup |f / c|$, we have

$$
L\left(v_{ \pm}\right)=c M \pm f \leqq 0 \quad \text { in } \bar{\Omega} .
$$

On the other hand, $v=$ may take a negative minimum value only on $\bar{\Sigma}_{+}$. In fact, suppose on the contraly, $v_{ \pm}$take a negative minimum value at some point $x_{0} \in \Omega$, it follows that $\partial v_{ \pm} / \partial x_{\imath}=0(i=1, \cdots, n)$ at $x_{0}$ and we have $L\left(v_{ \pm}\right)>0$ at $x_{0}$ since $v_{ \pm}\left(x_{0}\right)<0$. This contradicts (2.5). Similarly, we can show that any negative minimum value may be not taken at any $x_{0} \in \Sigma-\bar{\Sigma}_{+}$either. Since $v_{ \pm}=M \pm u=M \pm g \geqq 0$ on $\bar{\Sigma}_{+}$, we have $v_{ \pm} \geqq 0$ on $\bar{\Omega}$ and $\sup |u| \leqq M$. This proves the proposition.

Remark. In the above proposition, we cannot relax the condition $c(x)>0$ to $c(x) \geqq 0$.

EXAMPLE 2.4. Let $\Omega=\left\{(x, y) ; 1<x^{2}+y^{2}<4\right\}, \quad L u=y \frac{\partial u}{\partial x}-x \frac{\partial u}{\partial y}+c u=f$. Then all the boundary points belong to $\Sigma_{0}$. But for $c(x) \equiv 0$, all $u \equiv$ const. are solutions of (2.4). Note that in this case $\Gamma=\phi$.

In the following we consider our problem for $\Gamma=\bar{\Sigma}_{+}$or $\bar{\Sigma}_{-}$.

Proposition 2.4. Suppose $b(x) \neq 0$ on $\bar{\Omega}, \Sigma_{0}=\phi$. Further, suppose that there are no periodic orbits of (1.2) which are completely contained in $\bar{\Omega}$. Then for any $c(x) \in C^{\infty}(\bar{\Omega}),\left[W_{\infty}\right]$ is true when we choose $\Gamma=\bar{\Sigma}_{+}$or $\Gamma=\bar{\Sigma}_{-}$. 
The proof is trivial.

Remark. Conditions $\Sigma_{0}=\phi$ and the absence of periodic orbits are both essential for the validity of the proposition. We show this by the following examples.

ExAmple 2.5. (cf. Ex. 2.1.). For $L u=\frac{\partial u}{\partial x}$ and $\Omega=\left\{(x, y) ; 1<x^{2}+y^{2}<4\right\}$, $\left[W_{m}\right]$ is not true for $\Gamma=\bar{\Sigma}_{+}$and $\Gamma=\bar{\Sigma}_{-}$. Singularities of solutions may appear, for example, only on the line $y= \pm 1,0 \leqq x \leqq \sqrt{3}$ when $\Gamma=\bar{\Sigma}_{+}$.

EXAMPLE 2.6. Let $L u=a \frac{\partial u}{\partial x}+b \frac{\partial u}{\partial y}+c u=f$ and $\Omega=\left\{(x, y) ; 1 / 4<x^{2}+y^{2}\right.$ $<9 / 4\}$, where

$$
\begin{aligned}
& a=a(x, y)=x\left(x^{2}+y^{2}-1\right)-y\left(x^{2}+y^{2}+1\right), \\
& b=b(x, y)=y\left(x^{2}+y^{2}-1\right)+x\left(x^{2}+y^{2}+1\right) .
\end{aligned}
$$

Then

$$
\frac{d x}{d t}=a, \quad \frac{d y}{d t}=b
$$

has a periodic orbit $x^{2}+y^{2}=1$, and we see $\Sigma=\Sigma_{+}, \Sigma_{0}=\phi$ and $(a, b) \neq 0$ everywhere on $\bar{\Omega}$. But, for example, for $\Gamma=\bar{\Sigma}_{+},\left[W_{\infty}\right]$ is true if and only if

$$
\int_{0}^{2 \pi} c(\cos \theta, \sin \theta) d \theta \neq 0 \text {. }
$$

As the above example shows, the situation changes drastically if a periodic orbit exists. Suppose that there is a periodic orbit $x(t)$ of (1.2) which pass $x_{0} \in \bar{\Omega}$ at $t=0$ and has the minimal positive period $T$. Then, for any solution $u \in C^{m}(\bar{\Omega})$ of $L u=f$, we have (2.3) and since $U(T)=U(0)$ it must hold that

$$
U(0)\left(1-e^{h(T)}\right)=\int_{0}^{T} F(t) e^{h(t)} d t .
$$

Hence $L u=f$ has a smooth solution on this curve if and only if $e^{h(T)} \neq 0$, that is,

$$
\int_{0}^{T} C(t) d t=\int_{0}^{T} c(x(t)) d t \neq 0 .
$$

Further, then, the values $U(t)=u(x(t))$ on this curve are uniquely determined only by $f(x)$ without determining any initial data.

Proposition 2.5. Suppose $b(x) \neq 0$ on $\bar{\Omega}$, and $\Sigma=\Sigma_{+}$. Then, for $\Gamma=\Sigma_{+}$,

a) There is a sufficiently large positive number $c_{0}$ which depends on $b(x)$ and $m$ such that $\left[W_{m}\right]$ is true, for any $c \in C^{\infty}(\bar{\Omega})$ with $c(x) \leqq-c_{0}$, 
b) If $c(x)>0$ on $\bar{\Omega},\left[W_{m}\right]$ is not true.

Proof. a) is a consequence of a more general result which will be stated as Proposition 2.7. To prove b) we note that from the assumptions, any orbit $x(t)$ in $\bar{\Omega}$ through some $x_{0} \in \Sigma$ at $t=0$ has $\alpha$-limit set $C \subset \bar{\Omega}$ (see e.g. [4] §53.). Of course any orbit through a point $\in C$ is a periodic orbit in $\bar{\Omega}$. Then, for $f(x)=c(x)$, we have

$$
\begin{aligned}
U(t) & =e^{-h(t)}\left(U(0)+\int_{0}^{t} C(t) e^{h(t)} d t\right) \\
& =e^{-h(t)}\left(U(0)+\int_{0}^{t} \frac{d}{d t} e^{h(t)} d t\right) \\
& =e^{-h(t)}(U(0)-1)+1 .
\end{aligned}
$$

Hence if $c(x)>0$ on $\bar{\Omega}, h(t) \rightarrow-\infty$ as $t \rightarrow-\infty$ and thus $U(t) \rightarrow+\infty$ on this curve unless $U(0)=1$. This proves b).

Remark 1. Condition $c(x) \leqq-c_{0}$ in a) is not necessary for certain cases. It seems that we can relax the condition to $c(x)<0$ on certain parts of $\Omega$.

Remark 2. b) holds true when we only assume that $c(x)>0$ on at least one $\alpha$-limit set $C$ of the flows determined by (1.2).

ExAmple 2.7. Let $L u=y \frac{\partial u}{\partial x}-x \frac{\partial u}{\partial y}+c(x, y) u=f, \Omega=\left\{(x, y) ; 1<x^{2}+y^{2}<4\right\}$. Then $\Sigma=\Sigma_{0}$. We have that $\left[W_{m}\right]$ is true for $\Gamma=\phi$ if and only if

$$
\int_{0}^{2 \pi} c(r \cos t, r \sin t) d t \neq 0 \quad \text { for all } r \in[1,2] \text {. }
$$

(cf. Ex. 2.3. and $\S 3$ ) Especially $c(x) \neq 0$ on $\bar{\Omega}$ is sufficient.

For the example of b), see Example 2.6.

Next we consider the case when $\Sigma_{0} \neq \phi$. Then $\left[W_{m}\right]$ may not true even when $b(x) \neq 0$ on $\bar{\Omega}$ and ever any conditions on $c(x)$ are imposed.

ExAmple 2.8. Let $L u=\frac{\partial u}{\partial x}-4 x^{2} \frac{\partial u}{\partial y}+c u=f, \Omega=\left\{(x, y) ; x^{2}+y^{2}<1\right\}$. Then $\left[W_{m}\right]$ is not true and the singularity may appear only on the curve $y=-2 x^{2}+1$, $x \leqq 0$ for $\Gamma=\bar{\Sigma}_{+}$. (see also Ex. 2.5).

Proposition 2.6. Suppose $b(x) \neq 0$ on $\bar{\Omega}$ and further suppose that $\Sigma_{0}$ has no inner points and no periodic orbits contained in $\bar{\Omega}$ exist. Then for $\Gamma=\bar{\Sigma}_{+}$we have

a) If $\left[W_{m}\right]$ is true, then for any flow $x(t)$ through $x_{0} \in \Sigma_{0}$ at $t=0$, there is no nerghbourhood $\omega$ of $x_{0}$ such that $x(t) \in \Omega \cap \omega$ when $t$ is small.

b) If, for any $x_{0} \in \Sigma_{0}$, there is no nerghbourhood $\omega$ such that $x(t) \in \overline{\Omega \cap \omega}$ $-\left\{x_{0}\right\}$ when $t$ is small, then $\left[W_{m}\right]$ is valid. 
Proof. a) Suppose that there is a flow $x(t)$ through $x_{0} \in \Sigma_{0}$ at $t=0$ such that $x(t) \in \Omega \cap \omega$ for small $t$. Then from the continuity of solutions with respect to initial values and parameters and the fact that $\Sigma_{0}$ has no inner points, there is one parameter continuous family $x_{\varepsilon}$ of points on $\Sigma$ such that

$$
x_{0}=\left.x_{\varepsilon}\right|_{\varepsilon=0}, \quad x_{\varepsilon} \in \Sigma_{+} \text {when } \varepsilon>0, \quad x_{\varepsilon} \in \Sigma_{\text {- when }} \varepsilon<0 .
$$

The values of $u$ at $x_{\varepsilon}$ for $\varepsilon<0$ are determined by data given on a set $\sigma$ of points of $\bar{\Sigma}_{+}$, but since there are no periodic orbits, we see dist $\left(x_{0}, \sigma\right)>0$ and these values are independent of the initial values at $x_{\varepsilon} \in \Sigma_{+}, \varepsilon>0$. Thus there may exist a singular solution with singularities on the curve $x(t), t<0$, starting from $x_{0}$. This proves a).

b) Since $\Sigma_{0}$ has no inner points, we have, by integration, one and only one solution $u(x)$ for given boundary value $g$ on $\Gamma$. Now suppose that this solution have a singularity at some $x_{1} \in \Omega$, then $u$ must be singular at every point on the curve $x(t)$ through $x_{0}$. Since there are no periodic orbits and $b(x) \neq 0$, there are two end points $x_{+}, x_{-} \in \Sigma$ of $x=x(t), x_{+} \in \bar{\Sigma}_{+}, x_{-} \in \bar{\Sigma}_{-}$(cf. [4] Chap. 7). $x_{+} \in \Sigma_{+}$is impossible since the solution is smooth with respect to initial values and parameters. Thus it must be $x \in \Sigma_{0}$. But in this case, from the assumption, $x_{+}$must be an inner point of $\bar{\Sigma}_{+}$and $x_{+}$may not be a singular point. this proves b).

When we remove the condition $b(x) \neq 0$ on $\bar{\Omega}$, it seems that we cannot use the method of integration of the differential equation (1.2). We note the following result due to [2], [3] which is a restriction of more general result concerning second order equations.

Proposition 2.7. Suppose that $\Sigma_{+}$is closed in $\Sigma$. Then for any fixed $m$, there is a large positive constant $c_{0}$ such that for any $c(x) \in C^{\infty}(\bar{\Omega})$ with $c(x) \leqq-c_{0}$ on $\bar{\Omega},\left[W_{m}\right]$ is true for $\Gamma=\Sigma_{+}$. on $\bar{\Omega}$.

Remark. $c_{0}$ depends on the values of $b(x)$ and its second order derivatives

We omit the proof but refer to [2], [3].

COROLlary 2.8. Suppose that $\Sigma_{+}$is closed in $\Sigma$. If there is a function $\varphi \in C^{\infty}(\bar{\Omega})$ such that

$$
\sum_{\imath=1}^{n} b^{\imath} \frac{\partial \varphi}{\partial x_{\imath}} \neq 0 \quad \text { on } \quad \bar{\Omega} .
$$

then $\left[W_{\infty}\right]$ is valid for $\Gamma=\Sigma_{+}$and for any $c(x) \in C^{\infty}(\bar{\Omega})$.

Proof. Without loss of generality, we can assume that $\sum_{\imath=1}^{n} b^{\imath} \frac{\partial \varphi}{\partial x_{\imath}}<0$ on $\bar{\Omega}$. When we put $u=e^{\lambda \varphi} v$, the equation (1.1) is rewritten by

$$
\sum_{i=1}^{n} b^{\imath} \frac{\partial v}{\partial x_{\imath}}+\left(c+\lambda \sum_{i=1}^{n} b^{\imath} \frac{\partial \varphi}{\partial x_{\imath}}\right) v=f e^{-\lambda \varphi} \text {. }
$$


Thus if we take $\lambda>0$ sufficiently large such that $c+\lambda \sum_{\imath=1}^{n} b^{2} \frac{\partial \varphi}{\partial x_{\imath}}<-c_{0}$ on $\bar{\Omega}$, we can use Proposition 2.7.

For the existence of $\varphi \in C^{\infty}(\bar{\Omega})$ such that (2.6) is valid, it is necessary that $b(x) \neq 0$ on $\bar{\Omega}$. Further we must impose some topological conditions relating the integrability of (1.2) on $\bar{\Omega}$. For example we have

COROLlary 2.9 Suppose that $\Sigma_{+}$is closed in $\Sigma, \Omega$ is simply connected and

$$
\frac{\partial b^{k}}{\partial x_{\jmath}}=\frac{\partial b^{\jmath}}{\partial x_{k}}, \quad \jmath, k=1, \cdots, n, \quad b(x) \neq 0 \text { on } \bar{Q} .
$$

Then $\left[W_{\infty}\right]$ is true for $\Gamma=\Sigma_{+}$for any $c(x) \in C^{\infty}(\bar{\Omega})$.

Proof. The condition (2.7) is a integrability condition and it is well known that under this condition there is a function $\varphi \in C^{\infty}(\bar{\Omega})$ such that $\operatorname{grad} \varphi=b(x)$. For this $\varphi$, the condition (2.6) is valid.

Of course when the topological condition on $\Omega$ is not satisfied the conclusion is no longer true.

EXAMPLE 2.9. Let $L u=\frac{y}{x^{2}+y^{2}} \cdot \frac{\partial u}{\partial x}-\frac{x}{x^{2}+y^{2}} \cdot \frac{\partial u}{\partial y}+c u=f, \Omega=\{(x, y)$; $\left.1<x^{2}+y^{2}<4\right\}$. Then, except that $\Omega$ is simply connected, all the conditions of the corollary are satisfied. But as we have already seen in Example 2.7, $\left[W_{m}\right]$ is not true when

$$
\int_{0}^{2 \pi} c(r \cos t, r \sin t) d t=0
$$

for some $r \in[1,2]$.

\section{§3. Two dimensional case.}

As we have already seen, when we assume that $b(x)=0$ or $c(x) \leqq-c_{0}$ on $\bar{\Omega}$, certain conditions for the validity of $\left[W_{m}\right]$ are obtained. But in general, when both of these conditions are not valid, the general picture is not clarified yet.

In the following, we see some conditions for some simple equations. That is, we shall treat the equations

$$
L u=a \frac{\partial u}{\partial x}+b \frac{\partial u}{\partial y}+c u=f
$$

with

$$
\begin{gathered}
a(x, y)=p x+q y, \quad b(x, y)=r x+s y, \\
p, q, r, s \in \boldsymbol{R}, \quad p s-q r \neq 0 .
\end{gathered}
$$


We shall determine conditions of the validity of $\left[W_{m}\right]$ for this equation and for $\Omega \subset \boldsymbol{R}^{2}$ which contains the origin. We can generalize our results slightly by replacing the above $a, b$ with the following $\tilde{a}, \tilde{b}$ :

$$
\begin{aligned}
& \tilde{a}(x, y)=p x+q y+o(|x|+|y|), \\
& \tilde{b}(x, y)=r x+s y+o(|x|+|y|) .
\end{aligned}
$$

But the formulations are very complicated and we shall omit this case.

We first note two simple necessary conditions for $\Omega$.

Proposition 3.1. For the validity of $\left[W_{m}\right]$ for $\Gamma=\bar{\Sigma}_{+}$or $\Gamma=\Sigma_{-}$, it is necessary that

a) $\Omega$ is simply connected,

b) For any $P_{0} \in \Sigma_{0}$, the flow $P(t)=(x(t), y(t))$ through $P_{0}$ at $t=0$ satisfies the following condition

There are no interval $\left(t_{1}, t_{2}\right)\left(t_{1}<0<t_{2}\right)$ such that

$$
P(t) \in \bar{\Omega} \text { on }\left(t_{1}, t_{2}\right) \text { and } P\left(t_{1}\right), P\left(t_{2}\right) \in \Omega \text {. }
$$

Proof. We treat only the case $\Gamma=\bar{\Sigma}_{+}$. a) Suppose that there are compact components of $\boldsymbol{R}^{2}-\Omega$. Take $P_{0} \in \Sigma$ such that the flow $P(t)$ from $P_{0}$ intersects some bounded component $M$ of $\boldsymbol{R}^{2}-\Omega$ but does not intersect its inner points. Let $P_{1} \in M$ be a point where $P(t)$ first meets $M$ and $P_{2} \in M$ be a point where $P(t)$ separates from $M$. Then the value of $u$ on the curve $P(t)$, so especially the value $u\left(P_{2}\right)$ at $P=P_{2}$, are determined by the data given on the part of $\Sigma$ which are boundary points of unbounded component of $\boldsymbol{R}^{2}-\Omega$. On the other hand, since $P_{2} \in \bar{\Sigma}_{+} \cap \partial M$, the value $u\left(P_{2}\right)$ at $P=P_{2}$ is also determined by the data which are given arbitrary on $\bar{\Sigma}_{+} \cap \partial M$. This is a contradiction and this proves a). The proof of b) is a easy task.

Put

$$
A=\left[\begin{array}{ll}
p & q \\
r & s
\end{array}\right]
$$

and let $\lambda, \mu$ be eigenvalues of $A$. From the assumption $p s-q r \neq 0$, we have $\lambda \cdot \mu \neq 0$. Under these circumstances we prove the following theorem.

THEOREM 3.2. Let $\Omega \subset \boldsymbol{R}^{2}$ be a simply connected open set with $C^{\infty}$-boundary and $0 \in \Omega$. For the equation (3.1), (3.2), we assume the condition (3.3). Then

a) If $\lambda, \mu \in \boldsymbol{R}$ and $\lambda \cdot \mu>0$, then $\left[W_{m}\right]$ is valid for $\Gamma=\Sigma$ if and only if $c(0) / \max (\lambda, \mu)+m<0$. $\left[W_{\infty}\right]$ is valid for $\Gamma=\phi$ if and only if $\max (\lambda, \mu) \cdot c(0)>0$.

b) If $\lambda, \mu \notin \boldsymbol{R}$ and $\operatorname{Re} \lambda=\alpha \neq 0$, then $\left[W_{m}\right]$ is valid for $\Gamma=\Sigma$ if and only if $c(0) / \alpha+m<0$. [ $\left[W_{\infty}\right]$ is valid for $\Gamma=\phi$ if and only if $\alpha \cdot c(0)>0$.

c) If $\lambda, \mu \notin \boldsymbol{R}$ and $\operatorname{Re} \lambda=0$, then $\left[W_{\infty}\right]$ is valid for $\Gamma=\phi$ if $c(x, y) \neq 0$ on $\Omega$.

Proof. It is well known that by a real linear transformation of the coordinate systems we can reduce (3.1) to one of the following normal forms (see 
e.g. [4]):

$$
\begin{aligned}
& \lambda x u_{x}+\lambda y u_{y}+c u=f, \lambda \neq 0, \\
& \lambda x u_{x}+\mu y u_{y}+c u=f, \lambda \cdot \mu>0, \\
& \lambda x u_{x}+(k x+\lambda y) u_{y}+c u=f, \lambda \neq 0, k>0, \\
& (\alpha x+\beta y) u_{x}+(-\beta x+\alpha y) u_{y}+c u=f, \alpha \neq 0, \beta \neq 0, \\
& \beta y u_{x}-\beta x u_{y}+c u=f, \beta \neq 0, \\
& \lambda x u_{x}+\mu y u_{y}+c u=f, \lambda \cdot \mu<0 .
\end{aligned}
$$

Here and hereafter, we shall use the abridgements such as $u_{x}=\partial u / \partial x$, etc. Note that a) corresponds to the cases (I), (II) and (III), b) corresponds to (IV), c) corresponds to $(\mathrm{V})$. (For the case (VI) see the remark after the proof).

It is easy to see that when we prove the statements of the theorem for small disc $\omega \subset \Omega$ with center $0 \in \boldsymbol{R}^{2}$, the theorem follows for general $\Omega$. By streching the coordinates we can assume that $\omega=\left\{(x, y) ; x^{2}+y^{2}<1\right\}$. Thus it is sufficient to prove the theorem for this $\omega$ and for the normalized equations $(\mathrm{I}) \sim(\mathrm{V})$. Further we note that $c(0) \neq 0$ is a necessary condition for the validity of $\left[W_{m}\right]$. In fact, if $c(0)=0$, it must follow that $f(0)=0$ and $\left[W_{m}\right]$ is not true. Hence by streching the coordinates further, we may assume that $|c(x, y)| \geqq c_{0}>0$ on $\bar{\omega}$ for some positive constant $c_{0}$.

a) We shall consider the equation (I). Without loss of generality we may assume that $\lambda>0$. If we put

$$
x=e^{\lambda t} \cos \theta, \quad y=e^{\lambda t} \sin \theta,
$$

(I) can be written by

$$
\frac{d U}{d t}+C U=F
$$

where we used the notations $U=U(t, \theta)=u\left(e^{\lambda t} \cos \theta, e^{\lambda t} \sin \theta\right)$, etc. Thus the solution of (I) along the flow determined by (3.4) (which starts from a point on the circle $x^{2}+y^{2}=1$ at $t=0$ and tends to $(0,0)$ as $\left.t \rightarrow-\infty\right)$ can be written by

$$
u(x, y)=U(t, \theta)=e^{-h(t, \theta)}\left\{U(0, \theta)+\int_{0}^{t} F(t, \theta) e^{h(t, \theta)} d t\right\}
$$

where

$$
h(t, \theta)=\int_{0}^{t} C(t, \theta) d t=\int_{0}^{t} c\left(e^{\lambda t} \cos \theta, e^{\lambda t} \sin \theta\right) d t
$$

Especially

$$
v(x, y)=V(t, \theta)=e^{-h(t, \theta)} \int_{-\infty}^{t} F(t, \theta) e^{h\left(t \theta^{\theta}\right.} d t
$$


is a solution. We first prove that this solution is $C^{\infty}$ at $(x, y)=(0,0)$ as long as $c(0) / \lambda+m \neq 0(m=0,1,2, \cdots)$. When $c \geqq c_{0}>0$ on $\bar{\omega}, h \rightarrow-\infty$ as $t \rightarrow-\infty$ and when $c \leqq-c_{0}<0$ on $\bar{\omega}, h \rightarrow \infty$ as $t \rightarrow-\infty$. In both cases we can use the L'Hospital's theorem and have

$$
\lim _{t \rightarrow-\infty} V(t, \theta)=\lim _{t \rightarrow-\infty} e^{-h} \int_{-\infty}^{t} F e^{h} d t=\lim \frac{F e^{h}}{h^{\prime} e^{h}}=-\frac{f(0)}{c(0)} .
$$

Next, by differentiating (I) with respect to $x$ and $y$, we have

$$
\begin{aligned}
& (\lambda+c) u_{x}=f_{x}-c_{x} u-\lambda\left(x u_{x x}+y u_{x y}\right), \\
& (\lambda+c) u_{y}=f_{y}-c_{y} u-\lambda\left(x u_{x y}+y u_{y y}\right) .
\end{aligned}
$$

Hence, if we prove that $u_{x x}, u_{x y}, u_{y y}$ are bounded when $(x, y) \rightarrow(0,0)$ (the limits may depend on the path $(x, y) \rightarrow(0,0))$, we have that $u_{x}$ and $u_{y}$ have limits when $(x, y) \rightarrow(0,0)$ which are not depending on the path provided that $c(0)+\lambda \neq 1$. On the other hand, if $c(0)+\lambda=0$, then it follows that $f_{x}(0)-c_{x}(0) u(0)$ $=f_{x}(0)-c_{x}(0) f(0) / c(0)=0, f_{y}(0)-c_{y}(0) f(0) / c(0)=0$. This shows that compatibility conditions on $f$ at $(x, y)=(0,0)$ arise. By differentiating the equatios (I) successivly, we have compatibility conditions on $f$ when $c(0)+\lambda m=0(m=0,1,2, \cdots)$.

Now we shall prove that any derivatives of $V=V(t, \theta)$ with respect to $x$ and $y$ are bounded provided that $c(0)+\lambda m \neq 0$. Since $V_{x}=V_{t} t_{x}+V_{\theta} \theta_{x}=V_{t} \cos \theta$ $/ \lambda e^{\lambda t}+V_{\theta} \sin \theta / \lambda e^{\lambda t}$ we have to prove that $\lim _{t \rightarrow-\infty} V_{t} / e^{\lambda t}$ and $\lim _{t \rightarrow-\infty} V_{\theta} / e^{\lambda t}$ are bounded. If we note $V_{t}=-h_{t} V+F=-C V+F, V_{t t}=-C_{t} V-C V_{t}+F, C_{t}=c_{t}=c_{x} \lambda e^{\lambda t} \cos \theta$ $+c_{y} \lambda e^{\lambda t} \sin \theta$, etc., we have that the quantity

$$
\lim _{t \rightarrow-\infty} \frac{V_{\theta}}{e^{\lambda t}}=\lim _{t \rightarrow-\infty} \frac{1}{e^{\lambda t}}\left\{-h_{\theta} V+e^{-h} \int_{-\infty}^{t}\left(F_{\theta}+h_{\theta} F\right) e^{h} d t\right\}
$$

is bounded. On the other hand, from $\lim _{t \rightarrow-\infty} V_{t} / e^{\lambda t}=\lim _{t \rightarrow-\infty}(F-C V) / e^{\lambda t}=\lim _{t \rightarrow-\infty}\left(F_{t}-C_{t} V\right.$ $\left.-C V_{t}\right) / \lambda e^{\lambda . t}$, we have that the quantity

$$
\lim _{t \rightarrow-\infty} \frac{1}{e^{\lambda . t}}\left(1+\frac{c}{\lambda}\right) V_{t}=\lim _{t \rightarrow-\infty} \frac{F_{t}-C_{t} V}{\lambda e^{\lambda t}}
$$

is bounded. Thus $u_{x}$ is bounded as $(x, y) \rightarrow(0,0)$ if $1+c(0) / \lambda \neq 0$. Similarly we can prove that all the derivatives of $V$ with respect to $x$ and $y$ are bounded and hence $V \in C^{\infty}$ at $(x, y)=(0,0)$. When $c(0)>0$, by combining Proposition 2.3, the result stated in a) follows.

Now we assume that $c(0) \leqq-c_{0}<0$. Then by Proposition 2.3, we must prescribe data $g(x, y)=G(\theta)$ on $x^{2}+y^{2}=1$. Since we have a $C^{\infty}$-solution $V=v$ of (I) for any given $f$, we have only to prove the theorem when $f \equiv 0$. Then the solution with $\left.u\right|_{\Sigma}=g$ is given by

$$
u(x, y)=U(t, \theta)=e^{-h(t, \theta)} G(\theta) .
$$

We shall prove that when $c(0)+\lambda m<0$, the derivatives of $u=U$ with respect 
to $x$ and $y$ up to order $m$ are bounded. (In fact these tend to 0 as $t \rightarrow-\infty$ ). Since $U_{x}=\cos \theta \cdot U_{t} / \lambda e^{\lambda t}+\sin \theta \cdot U_{\theta} / \lambda e^{\lambda t}=e^{-h-\lambda t}\left\{-c \cdot \cos \theta \cdot G(\theta) / \lambda+\left(G_{\theta}(\theta)-h_{\theta} G(\theta)\right)\right.$ $\sin \theta / \lambda\}$ and since $h \rightarrow \infty$ as $t \rightarrow-\infty, U_{x}$ is bounded if and only if $-h-\lambda t$ are bounded as $t \rightarrow-\infty$ and hence if and only if $c(0) / \lambda+1<0$. By the similar arguments, we know that the derivatives of $U$ with respect to $x$ and $y$ up to order $m$ are bounded if and only if $c(0) / \lambda+m<0$, and then these derivatives are all tending to 0 as $(x, y) \rightarrow(0,0)$. Hence we have proved a) for (I). The statements for (II) and (III) are proved by similar arguments.

b) In this case, when we put

$$
x=e^{a t} \cos (\theta-\beta t), \quad y=e^{\alpha t} \sin (\theta-\beta t),
$$

we have

$$
u(x, y)=U(t, \theta)=e^{-h(t, \theta)}\left\{U(0, \theta)+\int_{0}^{t} F(t, \theta) e^{h(t, \theta)} d t\right\}
$$

along the flow defined by (3.6). That $v(x, y)=V(t, \theta)$ defined by (3.5) is $C^{\infty}$ at $(0,0)$ is proved by the same way as a). On the other hand, there are no compatibility conditions for $f$. In fact, by differentiating (IV) with respect to $x$ and $y$, we have

$$
\begin{aligned}
& (\alpha+c) u_{x}-\beta u_{y}=f-c_{x} u+(*), \\
& \beta u_{x}+(\alpha+c) u_{y}=f-c_{y} u+(* *),
\end{aligned}
$$

where $(*)$ and $(* *)$ are those terms which tend to 0 with $(x, y)$ provided that the second order derivatives of $u$ are bounded. Since

$$
\left|\begin{array}{cc}
\alpha+c & -\beta \\
\beta & \alpha+c
\end{array}\right|=(\alpha+c)^{2}+\beta^{2}>0
$$

no compatibility conditions may arise and the same is true for higher order derivatives. The rest of the proof is similar to that of a) and we omit it.

c) When we put

$$
x=r \cos \theta, \quad y=r \sin \theta,
$$

we have

$$
u(x, y)=U(r, \theta)=e^{-h(r, \theta)}\left\{U(r, 0)+\int_{0}^{\theta} F(r, \theta) e^{h(r, \theta)} d \theta\right\},
$$

where

$$
h(r, \theta)=\int_{0}^{\theta} C(r, \theta) d \theta=\int_{0}^{\theta} c(r \cos \theta, r \sin \theta) d \theta .
$$

By the periodicity condition on $U(r, \theta)$ with respect to $\theta$, we have

$$
U(r, 2 \pi)=U(r, 0)=e^{-h(r, 2 \pi)}\left\{U(r, 0)+\int_{0}^{2 \pi} F(r, \theta) e^{h(r, \theta)} d \theta\right\},
$$


and hence

$$
U(r, 0)=\left\{\int_{0}^{2 \pi} F(r, \theta) e^{h(r, \theta)} d \theta\right\} /\left(e^{h(r, 2 \pi)}-1\right)
$$

provided that $e^{h(r, 2 \pi)}-1 \neq 0$, that is,

$$
\int_{0}^{2 \pi} c(r \cos \theta, r \sin \theta) d \theta \neq 0
$$

Since $\lim _{r \rightarrow 0} U(r, 0)=f(0) / c(0)$, we have

$$
\lim _{r \rightarrow 0} U(r, \theta)=e^{-c(0) \theta}\left\{-\frac{f(0)}{c(0)}+\frac{f(0)}{c(0)}\left(1-e^{-c(0) \theta}\right)\right\}=\frac{f(0)}{c(0)} .
$$

Further, as in b), there arise no compatibility conditions of $f$ since e.g.

$$
\begin{aligned}
& c u_{x}+\beta u_{y}=f_{x}-c_{x} u+(*), \\
& -\beta u_{x}+c u_{y}=f_{y}-c_{y} u+(* *),
\end{aligned} \quad \begin{array}{rr}
c & \beta \\
-\beta & c
\end{array} \mid=c^{2}+\beta^{2}>0 .
$$

Thus we only have to prove that when $c \neq 0$ on $\bar{\omega}$, then all the derivatives of $U$ with respect to $x$ and $y$ are bounded as $(x, y) \rightarrow(0,0)$. By differentiating with respect to $x$, we have $U_{x}=-\sin \theta \cdot U_{\theta} / r+\cos \theta \cdot U_{r}$. It is clear that $\lim _{r \rightarrow 0} U_{r}$ is bounded and also $\lim _{r \rightarrow 0} U_{\theta} / r=\lim _{r \rightarrow 0}(-C U+F) / r=\lim _{r \rightarrow 0}\left(-C_{r} U-C U_{r}+F_{r}\right)$ is bounded. Thus $U_{x}$ is bounded as $(x, y) \rightarrow(0,0)$. Similarly, it can be shown that all the derivatives are bounded as $(x, y) \rightarrow(0,0)$ and thus we have completed the proof.

Remark. (VI) is the case in which the conditions $b(x, y)=(\lambda x, \mu y)$ vanishes at $(x, y)=(0,0)$ and $\Sigma_{0} \neq \phi$ are connected in a essential manner. In this case, it seems that we have to impose conditions on $c$ not only at $(x, y)=(0,0)$ but on some set in $\bar{\Omega}$. These cases, i. e. the case in which $b(x)=0$ and $\Sigma_{0} \cap \bar{\Sigma}_{+} \neq \phi$ $\left(\Sigma_{0} \cap \bar{\Sigma}_{-} \neq \phi\right)$ occur, will be treated in some general context in our subsequent note.

\section{$\S 4$. Examples of the first boundary value problem for second order equations.}

In the following, by using our preceding results, we shall present some examples of the first boundary value problem for second order equations. First we restate our problem which we have already stated in $\S 1$. For given second order equation

$$
L u=\sum_{\imath, \jmath=1}^{n} a^{\imath \jmath} \frac{\partial^{2} u}{\partial x_{i} \partial x_{\jmath}}+\sum_{\imath=1}^{n} b^{\imath} \frac{\partial u}{\partial x_{\imath}}+c u=f
$$

defined on $\bar{\Omega}$ with $a^{\imath \jmath}, b^{\imath}, c \in C^{\infty}(\bar{\Omega})$, find some closed set $\Gamma \leqq \Sigma=\partial \Omega$ such that the statement $\left[W_{m}\right]$ is true. 
It is well known that for elliptic equations, as the first boundary value problem (i. e. Dirichlet problem), $\left[W_{\infty}\right]$ is true for $\Gamma=\Sigma$ as long as min $\{-c(x)\}$ is sufficiently large positive number. Further, it is known that some kind of degenerate elliptic equations have this properties (see e.g. [2], [3]). This property is related to non-existence of non-degenerate characteristic curves. Thus we may expect that for the validity of $\left[W_{m}\right]$, it is necessary that

$$
\sum_{\imath, j=1}^{n} a^{\imath \jmath} \xi_{\imath} \xi_{j} \geqq 0 \quad \text { for all } \quad(x, \xi) \in \bar{\Omega} \times \boldsymbol{R}^{n} .
$$

But it is not true in general situation as we shall see by the following examples. Thus the problem "to investigate the relations between $L, \Omega, \Gamma$ under which $\left[W_{m}\right]$ is true" arises. We shall show some of the examples but shall not attempt to study such a problem in this note.

EXAMPLE 4.1. Let $\Omega=\left\{(x, y) ; x^{2}+y^{2}<1\right\}$ and

$$
\begin{aligned}
L & =y^{2} \frac{\partial^{2}}{\partial x^{2}}-2 x y \frac{\partial^{2}}{\partial x \partial y}+x^{2} \frac{\partial^{2}}{\partial y^{2}}+(\alpha y-x) \frac{\partial}{\partial x} \\
& +(-\alpha x-y) \frac{\partial}{\partial y}+\beta ; \alpha, \beta \in \boldsymbol{R} .
\end{aligned}
$$

It is clear that for the validity of $\left[W_{m}\right], \beta \neq 0$ is a necessary condition. Then

i) If $\beta<0,\left[W_{\infty}\right]$ is true for $\Gamma=\phi$,

ii) If $\beta>0$ and $\alpha^{2}>4 \beta,\left[W_{\infty}\right]$ is true for $\Gamma=\phi$.

In fact, by taking $c_{1}, c_{2} \in \boldsymbol{R}$ such that $c_{1}+c_{2}=\alpha, c_{1} c_{2}=\beta$, we have that

$$
L=L_{1} L_{2} \quad \text { where } \quad L_{j}=y-\frac{\partial}{\partial x}-x \frac{\partial}{\partial y}+c_{\jmath}, \quad \jmath=1,2 .
$$

Thus i), ii) follows from Theorem 3.2. By returning to the arguments of the proof, we have that for $\left[W_{\infty}\right]$ to be true it is not necessarily assumed that $c_{j} \in \boldsymbol{R}$. More precisely, when we consider complex valued function $c(x)$ in the arguments after Example 2.6, we have that we have only to assume that

$$
\int_{0}^{T} C(t) d t=\int_{0}^{T} c(x(t)) d t \neq 2 \pi k \imath, \quad k \in \boldsymbol{Z} .
$$

By applying this arguments we have that $\left[W_{\infty}\right]$ is true for (4.1) if and only if $c_{1}, c_{2} \neq k \imath, k \in Z$. For the simplicity we assume further $\alpha=0$ and consider the following equation;

$$
L=y^{2} \frac{\partial^{2}}{\partial x^{2}}-2 x y \frac{\partial^{2}}{\partial x \partial y}+x^{2} \frac{\partial}{\partial y^{2}}-x \frac{\partial}{\partial x}-y \frac{\partial}{\partial y}+\beta .
$$

Then we have that $\left[W_{\infty}\right]$ is true for (4.2) if and only if

$$
\beta \neq n^{2}, \quad n \in N \cup\{0\} .
$$


We can generalize this by assuming that $\beta$ is no longer constant. Then if there is a $C^{\infty}$-solution $c(x, y)$ of the equation

$$
y \frac{\partial c}{\partial x}+x \frac{\partial c}{\partial y}+c^{2}=-\beta !
$$

we can rewrite (4.2) by

$$
L=L_{+} L_{-} \quad \text { where } \quad L_{ \pm}=y \frac{\partial}{\partial x}-x \frac{\partial}{\partial y} \pm c .
$$

Thus, if we further assume that

$$
\int_{0}^{2 \pi} c(r \cos t, r \sin t) d t \neq 2 \pi k i, \quad r \leqq 1, \quad k \in \boldsymbol{Z},
$$

then $\left[W_{\infty}\right]$ is true. Note that if we attempt to find $c(x, y)$ by integrating ordinary differential equation

$$
\frac{d x}{d t}=y, \quad \frac{d y}{d t}=x,
$$

we have that $C(t)=c(x(t), y(t))$ must be a solution of Riccati's equation

$$
\frac{d C(t)}{d t}+C(t)^{2}=B(t)
$$

where $B(t)=\beta(x(t), y(t))$.

EXAmple 4.2 Let $\Omega$ be as above and

$$
\begin{aligned}
L= & x y \frac{\partial^{2}}{\partial x^{2}}+\left(y^{2}-x^{2}\right) \frac{\partial^{2}}{\partial x \partial y}-x y \frac{\partial^{2}}{\partial y^{2}}+(x-(c-1) y) \frac{\partial}{\partial x} \\
& +((c-1) x+y) \frac{\partial}{\partial y}-c ; c \in R .
\end{aligned}
$$

Then, for $\Gamma=\Sigma,\left[W_{m}\right]$ is true if and only if $m<c$ and for $\Gamma=\phi,\left[W_{\infty}\right]$ is true if and only if $c<0$. In fact $L$ can be written by

$$
L=L_{1} L_{2}, \quad L_{1}=y \frac{\partial}{\partial x}-x \frac{\partial}{\partial y}+1, \quad L_{2}=x \frac{\partial}{\partial x}+y \frac{\partial}{\partial y}-c,
$$

and the result follow from Theorem 3.2. Note that the above equation has no non-negative characteristic form.

EXAMPle 4.3. Let $\Omega$ be as above and

$$
L=x^{2} \frac{\partial^{2}}{\partial x^{2}}+2 x y \frac{\partial^{2}}{\partial x \partial y}+y^{2} \frac{\partial^{2}}{\partial y^{2}}+\alpha x \frac{\partial}{\partial x}+\alpha y \frac{\partial}{\partial y}+\beta ; \alpha, \beta \in \boldsymbol{R} .
$$

Then $\Gamma=\Sigma$,

i) If $\beta<0$ and $\alpha<-2 m+1$ or if $\beta<m(\alpha-1)-m^{2}$, then $\left[W_{m}\right]$ is true for 
BOUNDARY VALUE PROBLEMS FOR DIFFERENTIAL EQUATIONS

ii) If $\beta>0, \alpha>1$, then $\left[W_{\infty}\right]$ is true for $\Gamma=\phi$,

iii) If $\beta>0, \alpha<1$, then $\left[W_{m}\right]$ is not true for $\Gamma=\Sigma$ and $\Gamma=\phi$.

In fact, by taking $c_{1}, c_{2} \in \boldsymbol{R}$ such that $c_{1}+c_{2}=\alpha-1, c_{1} c_{2}=\beta$, we have

$$
L=L_{1} L_{2}, \quad L_{j}=x \frac{\partial}{\partial x}+y \frac{\partial}{\partial y^{\prime}}+c_{\jmath}, \quad \jmath=1,2,
$$

and thus the above result follow from Theorem 3.2. Especially by taking $\alpha=1$, we have the following result: For

$$
L=x^{2} \frac{\partial^{2}}{\partial x^{2}}+2 x y^{\prime} \frac{\partial^{2}}{\partial x \partial y}+y^{2} \frac{\partial^{2}}{\partial y^{2}}+x \frac{\partial}{\partial x}+y^{\prime} \frac{\partial}{\partial y}+\beta ; \beta \in \boldsymbol{R},
$$

$\left[W_{m}\right]$ is true if $\beta<-m^{2}$. (cf. Example 4.1 ).

ExAmple 4.4. Let

$$
\begin{aligned}
L= & y \frac{\partial^{2}}{\partial x^{2}}-(x+y) \frac{\partial^{2}}{\partial x \partial y}+x \frac{\partial^{2}}{\partial y^{2}}+\left(\alpha y^{\prime}+\beta\right)-\frac{\partial}{\partial x} \\
& -(\alpha x+\beta) \frac{\partial}{\partial y}+\alpha \beta ; \alpha, \beta \in \boldsymbol{R} .
\end{aligned}
$$

If $\Omega$ is as above, $\left[W_{\infty}\right]$ is valid for

$$
\Gamma=\left\{(x, y) ; x^{2}+y^{2}=1, x+y>0\right\} \quad \text { or } \quad \Gamma=\left\{(x, y) ; x^{2}+y^{2}=1, x+y<0\right\}
$$

if and only if $\beta \neq 0$. On the other hand, if we take

$$
\Omega=\left\{(x, y) ; 1<x^{2}+y^{2}<4\right\},
$$

then $\left[W_{m}\right]$ is not valid for any choice of $\alpha, \beta$ and $\Gamma$.

We hope to return to consider the problem stated at the begining of this section and related problems of propagations of singularities of solutions at a later time.

\section{REFERENCES}

[1] J. J. Duistermaat and L. Hörmander, Fourıer integral operators, II, Acta Math., 128 (1972), 183-269.

[2] J.J. Kohn And L. NirenberG, Degenerate elliptic-parabolic equations of second order, Comm. Pure Appl. Math., 20 (1967), 797-872.

[3j O. A. Oleinik and E. V. Radkevič, Second Order Equations With Nonnegative Characteristic Form, Moscow, 1971, English trans1., Amer. Math. Soc., 1973.

[4j I.G. Petrovski, Ordinary Differential Equatıons, Prentice-Hall, 1966. 